\title{
Évaluation de la résistance du palmier dattier au bayoud causé par Fusarium oxysporum f sp albedinis. Recherche d'une méthode de discrimination des vitroplants acclimatés en serre
}

\author{
My Hedra ${ }^{1 *}, \mathrm{M} \mathrm{Besri}^{2}$ \\ ${ }^{1}$ INRA, laboratoire de pathologie végétale-mycologie, \\ centre régional du Haouz-Présahara, BP 533, Marrakech \\ 2 Institut agronomique et vétérinaire Hassan-ll, département de phytiatrie, BP 6202, Rabat, Maroc
}

(Reçu le 12 novembre 1992 ; accepté le 3 septembre 1994)

\begin{abstract}
Résumé - Dans le programme de sélection du palmier dattier mené au Maroc contre le bayoud, les clones sélectionnés en palmeraie ou par croisements contrôlés ne sont représentés que par un seul à quelques individus. Les clones retenus de bonne qualité fruitière sont présumés résistants. Les tests de confirmation de leur résistance doivent être réalisés au stade plantule sur un nombre suffisant de vitroplants conformes et acclimatés en serre ; d'où la nécessité de mettre au point une méthode fiable de discrimination de ce type de matériel. À partir d'un isolat de Fusarium oxysporum $\mathrm{f} s p$ albedinis très agressif, et après comparaison de différentes concentrations d'inoculum du parasite, les concentrations d'inoculum $10^{4}$ et $10^{5}$ conidies par $\mathrm{ml}$ ont permis de discriminer les génotypes résistants et sensible à la maladie. Parmi 5 méthodes d'inoculation, la plus fiable consiste à apporter par plant $20 \mathrm{ml}$ de cet inoculum liquide ( $\left.10^{5} \mathrm{spores} / \mathrm{ml}\right)$ sur les racines de vitroplants élevés dans des sachets de polyéthylène, dénudées jusqu'à une profondeur de $5 \mathrm{~cm}$.
\end{abstract}

palmier dattier / bayoud / Fusarium oxysporum f sp albedinis / vitroplants / résistance

Summary - Evaluation of date palm resistance to bayoud disease due to Fusarium oxysporum $\mathrm{fsp}$ albedinis. Development of an efficient method for discriminating in vitro plantlets acclimatised in the glasshouse. In the Moroccan breeding program of the date palm to control bayoud disease, the clones selected in date palm groves from seedlings or by controlled breeding are only represented by one or few individuals. The clones selected on the criterion of fruit quality are presumed to be resistant to bayoud disease. The confirmation of resistance tests require a sufficient number of the identical in vitro plantlets in early stages and acclimatised in glasshouse. Consequently, it is necessary to develop an appropriate method for discriminating these plantlets. With a very agressive isolate of Fusarium oxysporum $f s p$ albedinis and after comparison of different inoculum concentrations of the pathogen, the 2 inoculum concentrations $10^{4}$ and $10^{5}$ conidia per $\mathrm{ml}$ allowed us to distinguish between the resistant and susceptible genotypes. Of 5 inoculation methods, the best was to apply $20 \mathrm{ml}$ per plant of such a liquid inoculum $\left(10^{5} \mathrm{spores} / \mathrm{ml}\right)$ to the roots of the plantlets at a depth of $5 \mathrm{~cm}$.

date-palm / bayoud /Fusarium oxysporum $f$ sp albedinis / in vitro plantlets / resistance 


\section{INTRODUCTION}

La fusariose vasculaire du palmier dattier (Phoenix dactylifera $L$ ) dénommée bayoud et causée par Fusarium oxysporum $\mathrm{f} \mathrm{sp}$ albedinis a fait des ravages considérables dans les palmeraies de l'Afrique du Nord. En effet, en l'espace d'un siècle, elle a détruit plus de 10 millions de palmiers au Maroc (Péreau-Leroy, 1958 ) et de 3 millions d'arbres en Algérie (Djerbi, 1988). Face à ce fléau qui constitue actuellement une menace potentielle pour les palmeraies limitrophes et les autres pays producteurs de dattes, des cultivars résistants de palmier dattier ont été sélectionnés (PéreauLeroy, 1958 ; Louvet et Toutain, 1973; Saaidi et al, 1981; Saaidi, 1992); ils sont malheureusement de qualité fruitière médiocre, ce qui explique la lenteur de leur diffusion.

Dans le programme de sélection des "Khalts", clones de palmier dattier issus de semis naturel, ou des plants de palmiers obtenus par croisements contrôlés, les génotypes choisis ne sont représentés que par un seul à quelques individus. Les tests de confirmation de leur résistance doivent être réalisés au stade plantule sur un nombre suffisant de vitroplants conformes et acclimatés en serre (Sedra, 1989a ; 1990), d'où la nécessité de mettre au point une méthode fiable de discrimination de ce type de matériel. La fiabilité des tests de discrimination dépend de la méthode d'inoculation et des conditions expérimentales. Dans la littérature, plusieurs techniques d'inoculation de jeunes plantules de palmier issues de graines ont été rapportées (Péreau-Leroy, 19857 ; Laville, 1962 ; Bulit et al, 1967 ; Renard et al, 1972 ; Louvet et Toutain, 1973, Dubost et Kada, 1974 ; Saaidi et al, 1981). À notre connaissance, aucune étude n'a été entreprise sur la résistance de vitroplants de palmier dattier élevés en serre visà-vis du bayoud.

Ce constat justifie que nous ayons cherché à mettre au point une méthode d'inoculation fiable, rapide et adaptée à ce type de matériel. Pour cela, nous nous sommes fixés 3 objectifs :

- sélectionner un isolat de $F$ of $\mathrm{sp}$ albedinis très agressif et parfaitement sélectif ;

- définir les concentrations d'inoculum du parasite permettant la discrimination entre le matériel sensible et résistant ;

- allier fiabilité des méthodes d'inoculation et simplicité d'utilisation.

\section{MATÉRIEL ET MÉTHODES}

\author{
Comparaison des niveaux d'agressivité \\ de quelques isolats de $\mathrm{F}$ of sp albedinis
}

L'agressivité de 5 isolats du parasite, issus de palmes atteintes prélevées sur différents cultivars plantés dans des régions éloignées (tableau I), est comparée. Pour chaque isolat, le niveau d'agressivité est apprécié sur 2 lots de plantules de palmier, âgées de 3 mois, élevées dans des sachets transparents en polyéthylène $(12 \mathrm{x}$ $25 \mathrm{~cm}$ ) et issues de 2 croisements réalisés entre parents sensibles (cv Jihel $x$ mâle S1) et résistants (cv Bousthami noire $x$ mâle NP3). La méthode d'inoculation des plantules au stade de 2 feuilles, consiste à apporter $10 \mathrm{ml}$ par plant d'une suspension de conidies du parasite $\left(10^{6}\right.$ conidies $/ \mathrm{ml}$ ) sur quelques racines à travers une perforation de la paroi du sachet en polyéthylène, le semis préalable des graines prégermées à la périphérie de chaque sachet facilitant ainsi l'inoculation des plantes et les observations ultérieures. Le substrat utilisé pour l'élevage des plantules est composé d'un mélange de sable et de tourbe (1v/2v). Quatre répétitions de 20 plantules sont utilisées par isolat et par croisement ; les essais sont conduits en printemps sous serre vitrée (température $22-33^{\circ} \mathrm{C}$, humidifié $70-80 \%$ ). Les niveaux d'agressivité des isolats sont appréciés tous les 15 j pendant 6 mois de culture par l'incidence de la maladie exprimée en pourcentage de plantes attaquées par le parasite.

\section{Étude de l'effet de la concentration d'inoculum}

Cette étude a été réalisée sur vitroplants de 3 cultivars sensibles Boufeggous (BFG), Bouskri (BSK) et Jihel (JHL), d'un cultivar résistant Saïrlayalate (SLY) et d'un clone $\mathrm{CH}-1$ sélectionné en palmeraie sur le critère de qualité fruitière et présumé résistant dans les tests menés au domaine de Zagora. Ces vitroplants ont été obtenus par la technique d'orgagogenèse, acclimatés en serre et élevés séparément dans des sachets individuels contenant un mélange terreux. Ils ont été inoculés au stade végétatif 2 ou 3 feuilles correspondant à l'âge d'un an, avec 6 concentrations d'inoculum de l'isolat Foa 133 (tableau I) : $0,10^{2}, 10^{3}, 10^{4}, 10^{5}$ et $10^{6}$ spores $/ \mathrm{ml}$ (essentiellement des microconidies). L'inoculation consiste à apporter $20 \mathrm{ml}$ d'inoculum liquide par plant sur les racines jusqu'à une profondeur de $5 \mathrm{~cm}$. L'incidence de la maladie sur les vitroplants inoculés a été estimée après 6 mois de culture sous serre sur 4 répétitions de 4 plants chacune par génotype et par concentration d'inoculum.

\section{Étude de la fiabilité de quelques méthodes d'inoculation des vitroplants}

\section{Cinq méthodes ont été comparées.}

I. Inoculum ( $10^{5}$ spores $/ \mathrm{ml}$ ) apporté sur les racines dans les conditions décrites ci-dessus. 
II. Inoculum injecté à l'aide d'une seringue fine au niveau de 2 racines principales par plant. Chaque plant reçoit 0,2 $\mathrm{ml}$ de l'inoculum (5 000 spores $/ \mathrm{ml}$ ).

III. L'injection de l'inoculum est effectuée au niveau du collet.

IV. Trempage des racines dans l'inoculum ( $10^{5}$ spores $/ \mathrm{ml}$ ) : après découpage du fond du sachet, les racines développées et enchevêtrées sont trempées au quart de la hauteur du sachet dans une suspension de spores pendant $15 \mathrm{~min}$. Les plants sont ensuite placés dans des bacs contenant un substrat infesté avec le parasite $\left(10^{5}\right.$ propagules $\left./ g\right)$. Ce substrat est composé d'un mélange de sable et de tourbe $(1 \mathrm{v} / 2 \mathrm{v})$. Ce mélange est trés réceptif au bayoud.

V. Les vitroplants sont plantés, sans trempage préalable dans le substrat infecté des bacs précités.

Selon les méthodes, les plants témoins reçoivent de l'eau ou sont cultivés sur le substrat non infesté.

Dans cette étude, nous avons utilisé des vitroplants au stade 2 feuilles appartenant à 2 génotypes sensibles (Boufeggous et Bouskri) ainsi qu'un cultivar résistant Saïrlayalate. Pour chaque méthode, 3 répétitions de 4 vitroplants chacune par génotype ont été utilisées. L'incidence de la maladie sur les vitroplants exprimée en pourcentage d'attaque a été notée après 6 mois de culture sous serre pour chaque méthode d'inoculation et chaque génotype.

Pour tous les essais, les notations sur le développement de la maladie ont été faites tous les 15 j pendant 6 mois. L'attaque des plantes dues au $F$ of sp albedinis a été confirmée par des réisolements du parasite effectués à partir des tissus atteints du collet, sur milieu de culture : extrait de malt gélosé.

Les résultats expérimentaux présentés sous forme de pourcentage de plants atteints par le parasite ont été comparés par analyse de la variance après transformation angulaire, suivie du test du Duncan.

\section{RÉSULTATS}

\section{Sélection d'isolats agressifs sur les plants de palmier dattier}

L'isolat Foa 133 est globalement plus agressif que les autres isolats sur les plantules issues des 2 croisements avec des pourcentages d'attaque très élevés atteignant $92 \%$ (tableau I). L'isolat Foa 16TB attaque davantage les plantules issues du croisement effectué entre parents sensibles qu'entre parents résistants. En revanche, l'isolat Foa 145 isolé du cultivar résistant Saïrlayalate est significativement le moins agressif sur les plantules issues du croisement effectué entre parents sensibles mais avec un niveau d'agressivité comparable sur les descendants des 2 croisements.

\section{Effet de la concentration d'inoculum sur l'évaluation de la résistance des vitroplants}

À la plus forte concentration d'inoculum (106 spores par $\mathrm{ml}$ ), les pourcentages d'attaque des vitroplants par le bayoud sont très élevés sur les cultivars sensibles Boufeggous, Bouskri et Jihel mais significativement différents de ceux obtenus sur le cultivar résistant Saïrlayalate et le clone $\mathrm{CH}-1$ présumé résistant (tableau II). Ces pourcentages dépassent $50 \%$ pour les 2 derniers génotypes. Cependant, il apparaît que les

Tableau I. Comparaison des niveaux d'agressivité de quelques isolats de Fusarium oxysporum f $\mathrm{sp}$ albedinis sur plantules de palmier issues de 2 croisements.

Isolat

Origine de prélèvement

$\begin{array}{llll} & \text { Cultivar } & \text { Région } & \text { Année } \\ & & & \\ \text { Foa } 133 & \text { Khalt } & \text { Tissergate } & 1985 \\ \text { Foa S2 } & \text { Boufeggous } & \text { M'hamid } & 1982 \\ \text { Foa } 5 Z & \text { Jihel } & \text { Zagora } & 1981 \\ \text { Foa } 145 & \text { Saïrlayalate* } & \text { Tata } & 1986 \\ \text { Foa } 16 \text { TB } & \text { Mejhoul } & \text { Tabouassamt } & 1981\end{array}$

Effet croisement

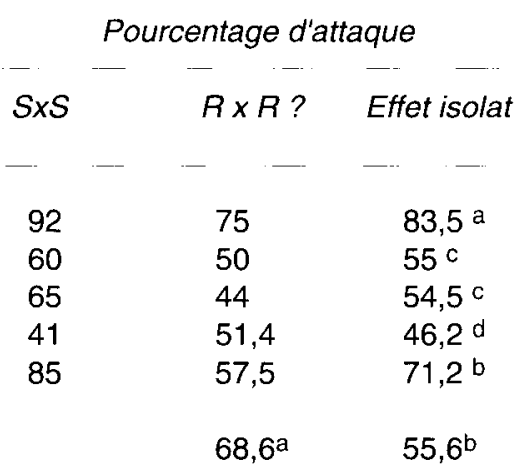

Pourcentages moyens d'attaque due au bayoud observés après 6 mois de culture sur les plantules issues de 2 croisements réalisés entre parents sensibles S $\times S$ (Jihel $\times$ ZS1) et parents résistants $R \times R$ (Bousthami noire $\times$ NP3). Ce pourcentage est calculé à partir de 4 répétitions de 20 plantes chacune par croisement et par isolat. Les chiffres suivis de la même lettre ne sont pas significativement différents pour $P=0,05$ (test de Duncan). $R$ ? = résistance du mâle non confirmée ; ${ }^{*}$ cultivar résistant. 
Tableau II. Effet de la concentration d'inoculum sur l'incidence du Fusarium oxysporum $\mathrm{f} s \mathrm{~s}$ albedinis sur les vitroplants de 4 cultivars et d'un clone de palmier.

Génotypes

Concentration d'inoculum (conidies $/ m$ l)

Effet

\begin{tabular}{|c|c|c|c|c|c|c|c|}
\hline & 0 & $10^{2}$ & $10^{3}$ & $10^{4}$ & $10^{5}$ & $10^{6}$ & génotypes \\
\hline Boufeggous (S) & 0 & 0 & $25^{b}$ & $43,7^{b}$ & $62,5^{\mathrm{a}}$ & 93,7 a & $37,5 \mathrm{AB}$ \\
\hline Bouskri (S) & 0 & 0 & $31,2^{a}$ & $56,2^{a}$ & $62,5^{a}$ & $100^{a}$ & $41,6 \mathrm{~A}$ \\
\hline Jihel (S) & 0 & 0 & $6,2^{\mathrm{c}}$ & $31,2^{c}$ & $50^{b}$ & $87,5^{\mathrm{a}}$ & $29,2^{B}$ \\
\hline Saïrlayalate (R) & 0 & 0 & $0 d$ & $12,5^{d}$ & $12,5^{c}$ & $62,5^{b}$ & $14,6^{C}$ \\
\hline Clone $\mathrm{CH}-1$ (R ?) & 0 & 0 & $0 d$ & $12,5^{d}$ & $18,7^{c}$ & $56,2^{b}$ & $14,6^{\mathrm{C}}$ \\
\hline Effet concentration d'inoculum & $\mathrm{OE}$ & $\mathrm{O}^{\mathrm{E}}$ & OD & $12,5^{\mathrm{D}}$ & $31,2^{C}$ & $41,2^{B}$ & $80^{A}$ \\
\hline
\end{tabular}

concentrations moyennes $\left(10^{4}\right.$ et $10^{5}$ spores par $\mathrm{ml}$ ) sont plus discriminantes entre les cultivars sensibles et les autres génotypes en provoquant plus ou moins d'attaque respectivement sur le matériel sensible ou résistant. En outre, une régression quadratique a été observée entre génotypes-concentrations d'inoculum. Ceci indique que la réponse des génotypes à l'augmentation de la concentration d'inoculum n'est pas linéaire.

\section{Comparaison de la fiabilité de différentes méthodes d'inoculation des vitroplants}

Les méthodes d'inoculation I, II, IV, V se révèlent fiables puisqu'elles permettent d'obtenir, après 6 mois de culture, des pourcentages d'attaque très élevés (> $60 \%$ en moyenne) sur le matériel génétique sensible mais relativement faibles sur le cultivar résistant Saïrlayalate (tableau III). Avec la méthode III (injection de l'inoculum au niveau

Tableau III. Influence de la méthode d'inoculation avec Fusarium oxysporum $\mathrm{f} s \mathrm{~s}$ albedinis sur l'incidence de la maladie.

Méthodes d'inoculation

Boufeggous

$75^{\star}$ a

$62,5^{a}$

$33,3 b$

$83,3^{\mathrm{a}}$

$75^{\mathrm{a}}$

$65,8^{A}$

Effet génotype
Génotypes

Bouskri

$83,3^{\text {a }}$

$75^{\mathrm{a}}$

41,7 a

$91,7^{\text {a }}$

$83,3^{\mathrm{a}}$

$75^{A}$
Effet méthode

Saïrlayate

$25^{\mathrm{b}}$
$50^{\mathrm{a}}$
$33,3^{\mathrm{b}}$
$58,3^{\mathrm{a}}$
$33,3^{\mathrm{b}}$

$61,1^{\mathrm{B}}$

$62,5 \mathrm{~B}$

$36,1^{\mathrm{C}}$

$77,8 \mathrm{~A}$

63,9 B

.

\footnotetext{
* Incidence de la maladie exprimée en pourcentage moyen de vitroplants atteints de la maladie calculé à partir de 3 répétitions de 4 plants chacune après 6 mois de culture sous serre. Les plants témoins utilisés pour chaque méthode ne présentent aucun signe de symptômes. Méthodes I, II, III, IV, et V (voir Matériel et méthodes). Les chiffres moyens suivis de la même lettre minuscule sur la même ligne ne sont pas significativement différents pour $p=0,05$ (test de Duncan). Aucune différence significative non plus n'a été révélée entre les chiffres moyens suivis de la même lettre majuscule. Régression quadratique entre génotypes-concentration d'inoculum.
} 


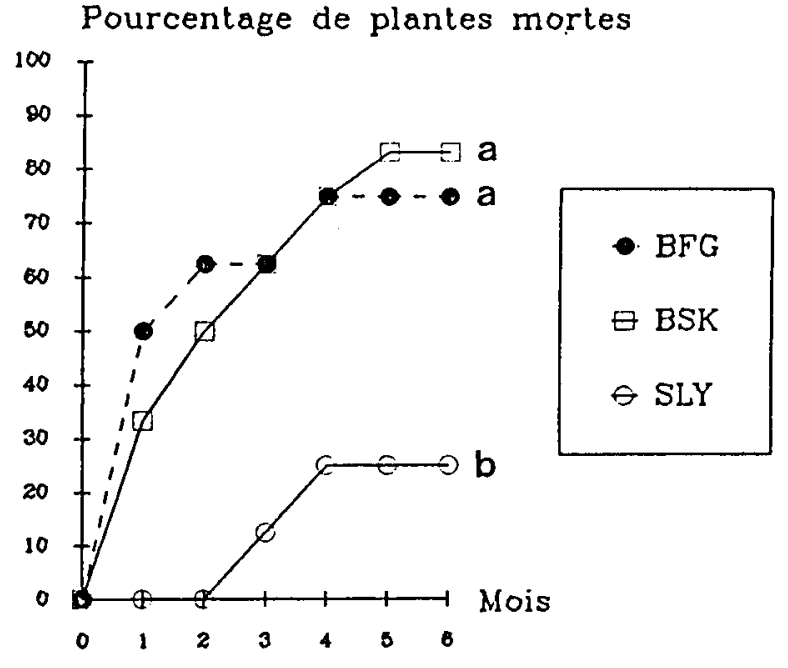

METHODE I

Fig 1. Incidence de la maladie due au Fusarium oxysporum $\mathrm{f}$ $\mathrm{sp}$ albedinis sur les vitroplants des cultivars sensibles (Boufeggous BFG, Bouskri BSK) et résistant (Saïrlayalate $S L Y$ ) en fonction du temps. Chaque point correspond au pourcentage moyen de vitroplants (inoculés avec la méthode "arrosage") atteints de la maladie calculé à partir de 3 répétitions de 4 plants chacune. Après 6 mois de culture, les points affectés d'une même lettre ne sont pas significativement différents pour $p=0,05$ (test de Duncan).

du collet des plantes), le pourcentage d'attaque des plants sensibles n'a pas dépassé $42 \%$. D'autre part, les méthodes I et $V$, en plus de leur fiabilité, permettent de façon significative la discrimination entre les cultivars sensibles et résistants. Les plants témoins utilisés pour chaque méthode n'ont manifesté aucun signe de flétrissement.

Par ailleurs, la figure 1 montre que le développement de la maladie en fonction du temps est différent entre les vitroplants de Saïlayalate et ceux de Boufeggous et Bouskri. En effet, le pourcentage d'attaque sur ces cultivars sensibles dépasse $30 \%$ seulement un mois, date à laquelle les premiers symptômes sur le cultivar résistant ont été observés ; mais, à partir du quatrième mois, le pourcentage d'attaque des plants résistants reste stable à $25 \%$.

\section{DISCUSSION}

Dans le premier essai, nous avons utilisé des plantules issues de semis faute de vitroplants. La méthode d'inoculation que nous avons appliquée, dans cet essai seulement, sur les descendants de croisements contrôlés pour apprécier les niveaux d'agressivité des isolats du parasite s'est révélée trés intéressante. Elle peut être aussi envisagée pour les tests des vitroplants. Mais, jusqu'à présent, nous ne disposions pas de vitroplants préalablement repiqués à la périphérie du sachet pour envisager l'application de cette méthode sur ce matériel.

La comparaison de l'agressivité des isolats de Fusarium oxysporum $\mathrm{f} s \mathrm{sp}$ albedinis étudiés fait apparaître une différence significative dans l'aptitude des isolats à attaquer des plants descendant des 2 croisements. L'isolat Foa 133 a été choisi pour la suite des expérimentations.

Les résultats obtenus sur les vitroplants montrent que la concentration de l'inoculum de $F$ of $\mathrm{sp}$ albedinis a un effet important sur l'incidence de la maladie dans les conditions expérimentales. Les concentrations discriminantes $\left(10^{4}\right.$ et $10^{5}$ spores $/ \mathrm{ml}$ ) permettent de différencier les cultivars sensibles (Boufeggous et Bouskri) du cultivar résistant Saïrlayalate. Dans une étude précédente, utilisant les descendants issus de plusieurs croisements différents de palmier dattier, nous avons démontré l'effet significatif de la concentration d'inoculum sur la résistance apparente des plantules (Sedra, 1989b). Cet effet a été également démontré dans le cas d'autres plantes par Douglas (1970) et Martyn et Mc Laughlin (1983). À la forte concentration d'inoculum $\left(10^{6}\right.$ spores $\left./ \mathrm{ml}\right)$, les pourcentages d'attaque, observés chez le cultivar résistant Saïlayalate et le clone $\mathrm{CH}-1$, présumé résistant, sont relativement élevés. À cette concentration, il apparaît que le matériel résistant testé est prédisposé, au stade plantule, à l'attaque du bayoud. Ceci nous a conduit à déterminer les concentrations discriminantes permettant de mettre en évidence une relation positive entre les comportements à l'égard du bayoud d'un matériel génétique donné aux stades adulte et jeune. Ces résultats obtenus sur des vitroplants acclimatés sont globalement comparables à ceux obtenus au champ par Saaidi (1992). À une concentration d'inoculum discriminante, le pourcentage d'attaque observé sur un matériel résistant peut varier de 12,5 à $18,7 \%$. Ceci est conforme aux observations de Martyn et Mc Laughlin (1983) pour la culture de la pastèque. Des recherches actuellement en cours devraient définir le stade végétatif des vitroplants au cours duquel le comportement à l'égard du bayoud est identique à celui des palmiers adultes. Pour le cultivar Saîrlayalate et le clone $\mathrm{CH}-1$, il semble que la résistance totale s'exprime avec l'âge puisque ces génotypes n'ont pas manifesté de maladie au stade adulte aprés inoculation expérimentale. 
La comparaison de la fiabilité des méthodes d'inoculation a révélé des différences : la "méthode arrosage" apparaît comme la mieux adaptée puisqu'en plus de son efficacité elle présente l'avantage de peu abîmer les racines au moment de l'inoculation et de provoquer précocement l'expression du maximum de symptômes sur les plants (résultats non présentés). Les résultats indiquent qu'après 2 mois et même seulement un mois les différences entre les niveaux d'attaque du matériel résistant et sensible sont aussi nettes qu'après 6 mois (fig 1). La «méthode trempage", qui présente une grande fiabilité, mérite d'être améliorée dans l'avenir.

\section{REMERCIEMENTS}

Nous remercions les techniciens du laboratoire de pathologie végétale (INRA-Marrakech) et particulièrement D Frira pour l'aide pratique qu'ils ont apportée pour réaliser ce travail. Nos remerciements vont également aux physiologistes qui ont produit les vitroplants, notamment le laboratoire de l'INRA-Marrakech et celui du domaine privé de Ouisslane MéknésMaroc. Nous remercions également la Fondation internationale pour la science (IFS) et le PNUD/FAO Projet Rab/88/024 pour leur support partiel au financement de ce travail.

\section{RÉFÉRENCES}

Bulit J, Louvet J, Bouhot D, Toutain G (1967) Recherches sur les fusarioses. I. Travaux sur le bayoud, fusariose vasculaire du palmier dattier en Afrique du Nord. Ann Epiphyties 18, 231-239

Djerbi M (1988) Les maladies du palmier dattier. Projet régional de lutte contre le bayoud. FAO (Rab/84/018), Alger, $127 \mathrm{p}$

Douglas DR (1970) The effect of inoculum concentration on the apparent resistance of muskmelons to Fusarium oxysporum $\mathrm{f} \mathrm{sp}$ melonis. Can J Bot 48, 687-693
Dubost D, A Kada (1974) Étude expérimentale de l'inoculation de jeunes palmiers dattiers issus de semis par Fusarium oxysporum $\mathrm{f} s \mathrm{p}$ albedinis. Bull Agr Sahar 1, 19-27 (CNRA, El Harrach-Algérie)

Laville E (1962) Infestation expérimentale de jeunes plantules du palmier dattier par Fusarium oxysporum var albedinis. Fruits 17, 88-90

Louvet J, Toutain G (1973) Recherches sur les fusarioses. VII. Nouvelles observations sur la fusariose du palmier dattier et précisions concernant la lutte. Ann Phytopathol 5, 35-52

Martyn RD, McLaughlin RJ (1983) Effects of inoculum concentration, on the apparent resistance of water melons to Fusarium oxysporum $\mathrm{f}$ sp niveum. Plant Disease 67, 493-495

Péreau-Leroy P (1957) Recherche d'un test de sensibilité des variétés de palmier dattier à la fusariose. Fruits 2, 12, 53-56

Péreau-Leroy $P$ (1958) Le palmier dattier au Maroc. Ministère de l'Agriculture, Instit Franc Rech Outremer, Paris, $142 \mathrm{p}$

Renard JL, Carton P, Bachi A (1972) Recherches sur la fusariose du palmier à huile. Oléagineux 27, 581-591

Saaidi M (1992) Comportement au champ de 32 cultivars de palmier dattier vis-à-vis du bayoud : 25 ans d'observations. agronomie 12, 259-270

Saaidi M, Toutain G, Bannerot H, Louvet J (1981) La sélection du palmier-dattier (Phoenix dactylifera $\mathrm{L}$ ) pour la résistance au bayoud. Fruits 4, 36, 241-249

Sedra My H (1989a) Sélection en palmeraie des palmiers résistants au bayoud et de bonne qualité du fruit. Méthodologie, résultats et problèmes. In : Sémin maghréb génét palmier dattier, 2-7 déc 1989, Adrar, Algérie, Doc FAO/INRA, Alger

Sedra My H (1989b) Étude de l'hérédité de la résistance au bayoud chez le palmier dattier. Intérêt de quelques facteurs à prendre en considération et étude de 3 séries de croisement dirigés. In : Sémin maghréb génet palmier-dattier, 2-7 déc 1989, Adrar, Algérie, Doc FAO/INRA, Alger

Sedra My H (1990) Preliminary results on the evaluation of the resistance to the bayoud of the clones (Khalts), cultivars and some hybrids of the date palm trees selected on the fruit quality criterium. In : 8th of Mediterr Phytopathol Union, 28 Oct - 3 Nov 1990, Agadir, Morocco 